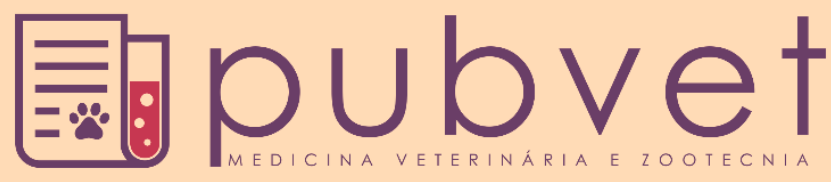

https://doi.org/10.31533/pubvet.v15n05a818.1-6

\title{
Coinfecção de anaplasmose e erliquiose: Relato de caso
}

\author{
Eduardo Junior Nascimento Sousa ${ }^{1 *}$, Natalia Lima Ferreira ${ }^{1}$, Maria Clara Fontenele de Sousa ${ }^{1} \bullet$, \\ Pollyana Oliveira da Silva ${ }^{2} \bullet$, Júlio Rodrigues Pereira Junior ${ }^{3} \bullet$, Márcio da Silva Costa $^{2}{ }^{\bullet}$, \\ Pâmela Christina Magalhães ${ }^{4}$ ๑
}

${ }^{I}$ Discente do curso de Medicina Veterinária da Universidade Federal do Piauí UFPI/CPCE, Brasil.

${ }^{2}$ Docente do curso de Bacharelado em Zootecnia da Universidade Federal do Piauí UFPI/CPCE, Brasil.

${ }^{3}$ Docente do curso de Medicina Veterinária da Universidade Federal do Piauí UFPI/CPCE, Brasil.

${ }^{4}$ Medica veterinária autônoma, Brasil.

*Autor para correspondência, E-mail: eduardo.jr.sousa@live.com

Resumo. A coinfecção de erliquiose e anaplasmose é muito comum na rotina clínica dos pequenos animais, são transmitidas principalmente pelo carrapato Rhipicephalus sanguineus, e provocam graves sinais clínicos, podendo evoluir a óbito. Foi realizado atendimento de um cão da Raça Labrador, macho, com 1,6 anos de idade, pesando $30 \mathrm{~kg}$, residente no município de Codó, no Estado do Maranhão, foi relatado na anamnese que permanecia apático, não estava se alimentando há alguns dias, com perda de peso e com manchas avermelhadas. No exame clínico o animal apresentou hipertermia de $42,5^{\circ} \mathrm{C}$, mucosas ocular e oral hipocoradas, inapetência, letargia, $8 \%$ de desidratação, tempo de preenchimento capilar (TPC) maior que 5 segundos, sensibilidade na região abdominal. Entretanto os batimentos cardíacos, movimentos respiratórios e linfonodos não apresentavam alterações. Para diagnóstico realizou-se teste rápido para Anaplasma e Ehrlichia no qual deram positivos, hemograma, bioquímico sérica hepática (AST e fosfatase alcalina) e renal (ureia) e urinálise. O resultado dos exames laboratoriais foi sugestivo ao diagnóstico de coinfecção por Anaplasma ssp. e Ehrlichia ssp. confirmando assim os resultados do teste rápido. Após o diagnostico iniciou-se o tratamento de suporte com fluidoterapia, complexo B, dexametasona, e como específico doxicilina e iminocard. O tratamento foi eficiente, o animal apresentou-se clinicamente saudável após 25 dia de tratamento.

Palavras-chave: Carrapato, cíclica, monocítica, pancitopenia, trombocitopenia

\section{Coinfection of anaplasmosis and ehrlichiosis: Case report}

Abstract. The ehrlichiosis and anaplasmosis' co-infection is awfully common to the clinical routine of small animals. They're mainly transmitted by the Rhipicephalus sanguineous tick and induce severe clinical symptoms, which are deadly able to lead specific animals to their death. A dog of the Labrador Breed was cared with 1.6-years-old male, weighing $30 \mathrm{~kg}$ and residing in Codó City, State of Maranhão, Brazil. In the anamnesis, it was reported that the dog was apathetic and without eat for few days, showing signs of weight loss and reddish spots. On clinical examination, the animal presented hyperthermia, pale and ocular mucous membranes, lethargic ineptitude, $8 \%$ of dehydration and capillary filling time greater than 5 seconds. Nevertheless, the animal doesn't showed changes in the heart rate, breathing movements and lymph nodes. For diagnosis, a rapid test was performed for Anaplasma and Ehrlichia in which were positive. Complete blood count, hepatic and renal serum biochemistry and urinalysis were also applied. The laboratory exams' results were suggestive for the diagnosis of co-infection by Anaplasma spp. and Ehrlichia spp, confirming the rapid test results. After diagnosis, supportive 
treatment with fluid therapy, B complex, dexamethasone, doxicillin and iminocard was started. The treatment was efficient. Therefore, the animal was clinically healthy after 25 days of treatment.

Keywords: Tick, cyclic, monocyclic, pancytopenia, thrombocytopenia

\section{Coinfección de anaplasmosis y ehrlichiosis: Reporte de caso}

Resumen. La infección de ehrlichiosis y anaplasmosis es muy común en la rutina clínica de los animales pequeños, son transmitidos en general por las garrapatas Rhipicephalus sanguineus, y generan graves indicios clínicos, y puede evolucionar hacia la muerte del animal. Se realizó la asistencia a un perro de raza Labrador, de sexo masculino, con 1.6 años de edad, con un peso de $30 \mathrm{~kg}$, el cual reside en el municipio de Codó, en el Estado de Maranhão, y fue reportado en la anamnesia que estaba apático, no había comido bien desde algunos días, y esto causó pérdida de peso y con puntos rojos, en el examen clínico el animal reveló hipertermia de $42.5^{\circ} \mathrm{C}$, membranas mucosas oculares y blanqueado oral, inapetencia, letargo, $8 \%$ de deshidratación, tiempo de llenado capilar mayor a 5 segundos, pero presentó los latidos del corazón, movimientos respiratorios y ganglios linfáticos sin alteraciones. El animal también mostró sensibilidad en la región del abdomen. Para el diagnóstico se efectuó una prueba rápida para Anaplasma y Ehrlichia en los que aprobaron positivos, conteo de glóbulos, bioquímico de suero hepático (AST y fosfatasa alcalina) y renal (urea) y examen de orina. El resultado de los exámenes laborales fue sugestivo al diagnóstico de coinfección por Anaplasma ssp. y Ehrlichia ssp. confirmándose así los resultados de la prueba rápida. Después del diagnóstico, empezó el tratamiento de soporte con terapia de fluidos, complejo $\mathrm{B}$, dexametasona, y como principal la doxiciclina e iminocard. El tratamiento fue eficaz, el animal se exhibió clínicamente saludable 25 días del inicio del tratamiento.

Palabras clave: Garrapatas, cíclico, monolítico, pancitopenia, trombocitopenia

\section{Introdução}

A Erliquiose e Anaplasmose são doenças infectocontagiosa causada por bactérias intracelulares hematopoiéticas obrigatórias, gram-negativas pertencentes à ordem Rickettssiales, família Anaplasmataceae, gêneros Erhlichia e Anasplasma, respectivamente (Garcia et al., 2018). São doenças transmitidas ao hospedeiro pelo vetor Rhipicephalus sanguineus, ou por transfusões sanguíneas (Sousa, 2006). Por se adaptar em diversos climas e regiões o carrapato apresenta distribuição mundial, o que torna sua presença constante nos animais de companhia (Dantas-Torres, 2010). Ainda, segundo o autor, sua presença tem aumentado a frequência de infecções por hemoparasitas. Em estudo realizado por Cetinkaya et al. (2016) pode se observar a presença de ambos os agentes etiológicos (Erhlichia e Anasplasma) em carrapatos, o que explica a ocorrência de hospedeiros coinfectados.

A prevalência de coinfecções entre Erhlichia e Anaplasma tem sido relatada em 9,1\% de cães sintomáticos através de testes rápidos de rotina (Peixoto, 2019) e em 24\% com uso de diagnóstico molecular (Costa et al., 2015). Em pacientes portadores de leishmaniose canina a prevalência desta coinfecção passa para 22\% dos casos diagnosticado por teste molecular (Costa et al., 2015).

Os principais sinais clínicos encontrados nas coinfecções por Erhlichia e Anaplasma são anemia e alterações oftalmológicas, associado a trombocitopenia (Peixoto, 2019). Essas alterações oftalmológicas estariam associadas ao gênero Erhlichia, pois não foi diagnosticado nenhum animal que tivesse apenas Anaplasma com essas alterações. Entre as alterações bioquímicas são relatadas aumento da concentração de globulinas e redução na concentração sérica de albumina, além do aumento nas enzimas hepáticas como Alanina aminotransferase (ALT) e Aspartato aminotransferase (AST) (Costa et al., 2015).

O objetivo do estudo foi relatar um caso de coinfecção por Erhlichia e Anaplasma em um cão. 


\section{Relato de caso}

Foi atendido um cão da raça Labrador, macho, com 1,6 anos de idade, pesando $30 \mathrm{~kg}$, na cidade de Codó, no Estado do Maranhão. Na anamnese foi relatado que o animal estava apático, não estava se alimentando há alguns dias, com perda de peso e com manchas vermelhas (Figura 1) na região da barriga e patas. A tutora também informou a presença de carrapatos dias antes no animal, mas que após banho com shampoo carrapaticida não havia mais visualizado as mesmas.

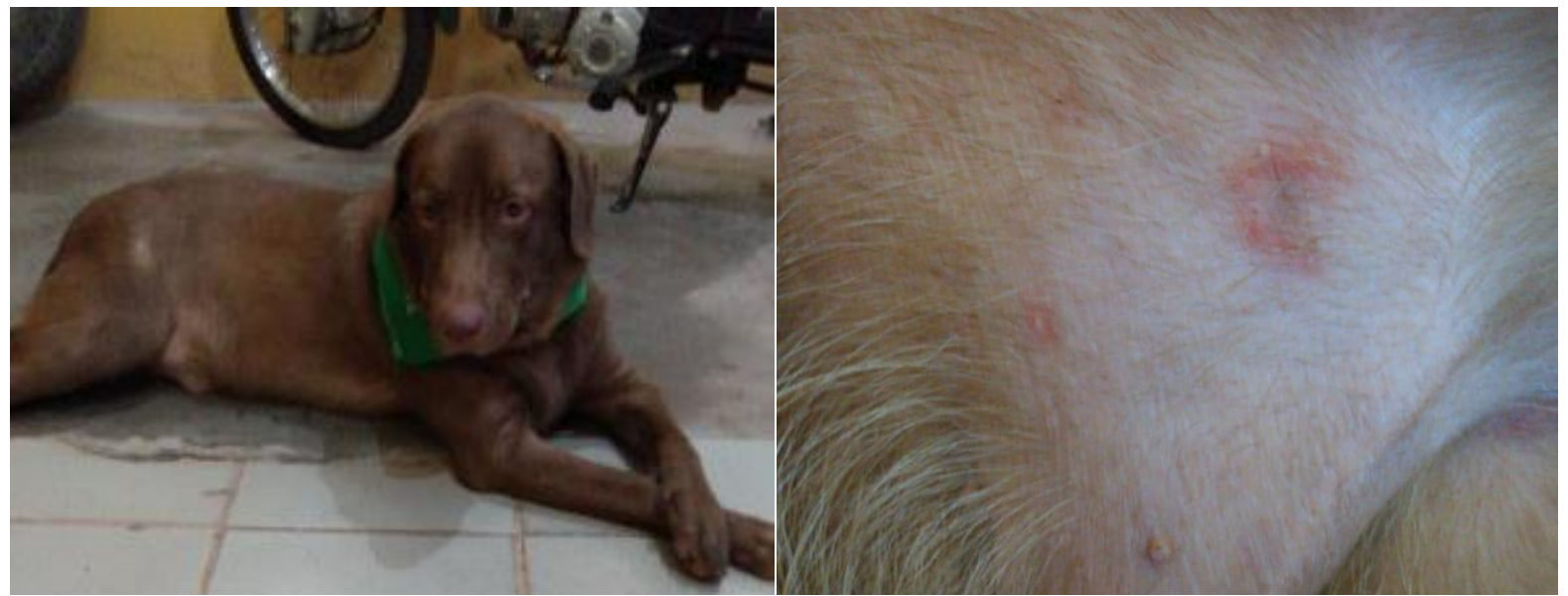

Figura 1. Paciente cão da raça Labrador, macho, de 1,6 anos de idade, com suspeita de infecção por hemoparasitas. Apresentando manchas avermelhadas presente na região inguinal.

Durante o exame clínico verificou que o animal apresentava hipertermia com temperatura de $42,5^{\circ}$ C, mucosas ocular e oral hipocoradas, inapetência, letargia, sinais de perda de peso, $8 \%$ de desidratação, tempo de preenchimento capilar (TPC) maior que 5 segundos, entretanto com parâmetros cardiorrespiratórios normais e linfonodos sem alteração. Ainda no exame clinico apresentou sensibilidade abdominal e visualização de manchas avermelhadas na região ventral e também na região femoral externa. No exame clinico não foi observado a presença de ectoparasitas. Com os achados clinicos a suspeita foi de Erliquiose ou Leishimaniose canina.

Para o diagnóstico foi coletada amostras para a realização dos exames complementares: hemograma, bioquímico sérica hepática (AST e fosfatase alcalina) e renal (uréia), urinálise, teste rápido para leshimaniose (Alere Leishimaniose Ac test Kit, Brasil ${ }^{\circledR}$ ), Dirofilária immitis, Anaplasma phagocytophilum, Anaplasma platys, Borrelia burgdorferi, Ehrlichia canis e Ehrlichia ewingii (SNAP 4Dx Plus IDEXX Laboratories ${ }^{\circledR}$, EUA). Após a realização dos testes rápidos obteve-se o diagnóstico para Anaplasma e Ehrlichia.

Com o diagnóstico de coinfecção Anaplasma e Ehrlichia inicialmente instituiu um tratamento de suporte através de fluidoterapia com um litro de solução de cloreto de sódio a $0,9 \%$ (33ml $/ \mathrm{kg} / \mathrm{h})$, dipirona na dose de $25 \mathrm{mg} / \mathrm{kg}$ a cada 8 horas, por 5 dias e vitamina do complexo B. Como tratamento específico para hemoparasitose foi administrado Doxiciclina $(6,7 \mathrm{mg} / \mathrm{kg})$ por 21 dias e Iminocard $(5 \mathrm{mg} / \mathrm{kg})$.

\section{Resultados e discussão}

Os achados clínicos levaram a suspeita de infecção por hemoparasitas, que foi confirmada com os exames complementares. O teste imune ensaio cromatográfico Alere Leishimaniose Ac teste $\mathrm{Kit}^{\circledR}$ apresentou resultado negativo, o que descartou a suspeita de leishmaniose canina. Já o teste SNAP 4DX PLUS, apresentou resultado positivo para Anaplasma ssp. e Ehrchia ssp, o que levou a um diagnóstico de coinfecção. Segundo Fonseca (2012), a observação do vetor no animal em conjunto com as manifestações clínicas, reforça a suspeita peculiares das doenças, portando a utilização de testes rápidos para hemoparasitose é recomendado para auxiliar no diagnóstico. Souza et al. (2012) ainda afirmam que a associação dos resultados hematológicos e sinais clínicos, são os métodos mais utilizados para diagnóstico de hemoparasitose.

Os sinais clínicos apresentados pelo paciente como anorexia, apatia e equimoses, apesar de não serem especificas, são decorrentes das diversas alterações fisiológicas que esses hemoparasitas causam no 
animal. A maioria deles por consequência da alteração de homeostase, quer seja por uma hipoplasia medular, destruição dos hemocomponentes, ou lesão vascular. Esses achados estão em acordo com a maioria dos sinais clínicos descrito por Silva et al. (2015) e Holanda et al. (2019)), que observaram apatia em $41,5 \%$ dos casos e anorexia em $26 \%$. A presença de petéquias no abdômen foi um sinal também descrito por Sá et al. (2018).

Os achados hematológicos (Tabela 1) apresentaram anemia normocítica normocrômica, com observação de anisocitose e policromasia discretas, além de trombocitopenia. Sousa (2006) notou em 27 cães infectados por $E$. Canis e A. platys, anemia normocítica normocrômica, o que vai de acordo com os encontrados. Já em estudo feito por Mota et al. (2019) com 113 cães considerados portadores de hemoparasitoses além da anemia e trombocitopenia, e também foi observado leucopenia. A anemia pode ser decorrente da remoção de eritrócitos da circulação pelo sistema mononuclear fagocítico e lise pelo sistema complemento, além da aplasia da medula óssea (Santos et al., 2019) e da deposição de imunocomplexos nos rins, onde causa redução eritropoietina sérica e consequentemente da eritropoiese (Silva et al., 2015).

Tabela 1. Resultados do Hemograma e Bioquímico renal e hepática de um canino da raça Labrador, macho,1,6 anos de idade, com coinfecção de Erhlichia e Anaplasma.

\begin{tabular}{lcc}
\hline Hemograma & Valor & Valor de Referência \\
\hline Hemácias & $4,10 \mathrm{mi} / \mathrm{mm}^{3}$ & $5,5-8,5$ \\
Hemoglobina & $10,1 \mathrm{~g} / \mathrm{dL}$ & $12,0-18,0$ \\
Hematócrito & $30 \%$ & $37-55$ \\
V.G.M. & $72,20 \mathrm{fL}$ & $60-77$ \\
C.H.G.M & $34,12 \mathrm{~g} / \mathrm{dL}$ & $30-36$ \\
R.D.W. & $12,2 \%$ & $12-17$ \\
Proteína Plasmática. Total & $5,6 \mathrm{~d} / \mathrm{dL}$ & $5,5-8,0$ \\
Eritroblastos & $0 \%$ & $0-5$ \\
Leucócitos & $12.000 / \mathrm{uL}$ & $6.000-17.000$ \\
Mielócitos & 0 & $0-0$ \\
Metamielócitos & 0 & $0-0$ \\
Bastonetes & 0 & $0-300$ \\
Segmentados & $9.317 / \mathrm{uL}$ & $3.000-11.500$ \\
Eosinófilos & $242 / \mathrm{uL}$ & $100-1.250$ \\
Basófilos & 0 & Raros \\
Monócitos & $847 / \mathrm{uL}$ & $150-1350$ \\
Linfócitos & $1.694 / \mathrm{uL}$ & $1.000-4.800$ \\
Plaquetas & $81.000 / \mathrm{mm}$ & $175.000-500.000$ \\
\hline Bioquímico & Valor & Valor de Referência \\
\hline Alanina Aminotransferase & $343 \mathrm{U} / \mathrm{L}$ & $10-88$ \\
Creatinina & $1,0 \mathrm{mg} / \mathrm{dL}$ & $0,5-1,5$ \\
Fosfatase Alcalina & $351 \mathrm{U} / \mathrm{L}$ & $0-156$ \\
Ureia & $54 \mathrm{mg} / \mathrm{dL}$ & $10-60$ \\
\hline
\end{tabular}

A trombocitopenia ocorre em virtude do aumento da destruição plaquetária causada por ambas as bactérias, essa alteração foi observado por outros autores, como Holanda et al. (2019) que observaram trombocitopenia em mais de $97 \%$ dos cães positivos para Ehrchilia ssp. e hipoplasia medular em 50\% dos positivos para Anaplasma spp., além da hiperplasia da série eritroide.

Nos achados bioquímicos, destacou-se elevação das enzimas (ALT) e Fosfatase Alcalina, tal fato pode ser decorrente de dano hepático e estresse sistêmico provocado pelo parasitismo (Gomes et al., $\underline{2008}$ ). Em estudo realizado por Costa et al. (2015) também observou alterações nas médias de ALT, AST e/ou FA, para animais com coinfecção por Erhlichia e Anasplasma. Neste caso, além do fator infecção por hemoparasitas temos a administração de corticoides anteriormente, pode ter causando uma degeneração dos hepatócitos promovendo elevação dos níveis séricos dessas enzimas (Pereira et al., 2011). Essas alterações, ainda explicam a sensibilidade à palpação na região abdominal, pois pode ser decorrente de alterações hepáticas, provavelmente um aumento esplênico ou hepático. De acordo com Lasta (2011) as plaquetas infectadas são removidas da circulação por sequestro esplênico, podendo causar uma esplenomegalia o que explica a sensibilidade abdominal. 
No exame de urinálise observou-se volume reduzido, densidade aumentada, presença de proteínas, além da presença de cristais de bilirrubina e aumento de bactérias. Com a desidratação, ocasionada pela redução da ingestão de líquidos, houve a redução do volume da urina, e com isso um aumento nos demais parâmetros. Entretanto o aumento de proteínas e bilirrubina são indícios da consequência de deposição de imuno-complexos nos rins e lesão hepática respectivamente (Silva et al., 2015).

Após o diagnóstico foi iniciado o tratamento de suporte com a administração de fluidoterapia solução de cloreto de sódio a $0,9 \%$, com 1 ampola de complexo B12, nos primeiros 500ml, e Dexametasona $0,15 \mathrm{mg} / \mathrm{kg}$, nos $500 \mathrm{ml}$ restante. Foi prescrito como tratamento com Doxiclina 200mg, BID, por 21 dias, via oral, associado a ranitidina $60 \mathrm{mg}$, BID, via oral, 30 minutos antes, suplemento alimentar (hemolitan gold $^{\circledR}$ ) 1 comprimido a cada $10 \mathrm{~kg}$, SID, Via oral, por 30 dias e silimarina $20 \mathrm{mg} / \mathrm{kg}$, SID, via oral, por 21 dias. Após 10 dias de tratamento foi adicionado ao protocolo iminocard $5 \mathrm{mg} / \mathrm{kg}$, via subcutânea, com Sulfato de Atropina 15 minutos antes, e repetida a dose com sete dias.

O tratamento de suporte foi realizado com soro para corrigir a desidratação, e adicionado o complexo B12 no intuito de melhorar quadro anemia. Para corrigir a trombocitopenia foi administrado a dexametasona, realizado com base nos estudos de Kastelan et al. (2009) que relaciona os corticoides ao aumento de plaquetas, pois a terapia imunossupressora com corticosteroides estabiliza o endotélio vascular, diminuindo a fagocitose das plaquetas pelos macrófagos, causando assim um possível aumento da produção de plaquetas. Foi utilizado como tratamento específico para hemoparasitose doxiciclina por 21 dias e iminocard, como descrito por Segundo Damas (2012), entretanto um fator limitante no tratamento é o parasitismo intraplaquetário, que dificulta ação dos fármacos.

No vigésimo quinto dia após o início do tratamento, retornou-se ao domicilio para uma nova avaliação clínica do animal, que se apresentou com todos os seus parâmetros dentro da normalidade. A Anaplasmose e Erliquiose são doenças muito recorrentes na clínica veterinária nos dias de hoje e sua coinfecção vem crescendo cada vez mais, sendo necessário sempre explorar a difusão de novos conhecimentos técnicos e científicos para concretizar a melhor forma de reconhecer essas doenças, quer seja por sinais clínicos, hematológicos ou específicos.

\section{Referências}

Cetinkaya, H., Matur, E., Akyazi, I., Ekiz, E. E., Aydin, L., \& Toparlak, M. (2016). Serological and molecular investigation of Ehrlichia spp. and Anaplasma spp. in ticks and blood of dogs, in the Thrace Region of Turkey. Ticks and Tick-Borne Diseases, 7(5), 706-714. https://doi.org/10.1016/j.ttbdis.2016.02.021.

Costa, M. P., Horta, R. S., Coura, F. M., Mol, J. P. S., Valente, P. C. L. G., \& Paes, P. R. O. (2015). Bioquímica sérica de cães infectados por Ehrlichia canis, Anaplasma platys e Leishmania sp. Acta Scientiae Veterinariae, 43, 1-7.

Damas, J. K. A. (2012). Erliquiose Canina: Revisão de Literatura. Universidade Paulista.

Dantas-Torres, F. (2010). Biology and ecology of the brown dog tick, Rhipicephalus sanguineus. Parasites \& Vectors, 3(1), 26. https://doi.org/https://doi.org/10.1186/1756-3305-3-26.

Fonseca, J. P. (2012). Erliquiose canina em lavras, sul de minas gerais, Brasil. In Dissertação. Universidade Federal de Lavras.

Garcia, D. A., Martins, K. P., Cortezi, A. M., \& Gomes, D. E. (2018). Erliquiose e Anaplasmose CaninaRevisão de Literatura. Revista Científica, 1(1).

Gomes, A., Parra, B.S., Franco, F.O., Basile, L., Jose, L.T., Romero, V.L. \& Sacco, S.R. (2008). Exame da função hepática na Medicina Veterinária. Revista Científica Eletrônica de Medicina Veterinária, 11(2): 1-7.

Holanda, L. C., Almeida, T. L. A. C., Mesquita, R. M., Oliveira Júnior, M. B., \& Oliveira, A. A. F. (2019). Achados hematológios em sangue e medula óssea de cães naturalmente infectados porEhrlichia spp. E Anaplasma spp. Ciência Animal Brasileira, 20, 1-12. https://doi.org/10.1590/1809-6891v20e-47686

Kastelan, D., Dusek, T., Kraljevic, I., Polasek, O., Giljevic, Z., Solak, M., Salek, S. Z., Jelcic, J., Aganovic, I., \& Korsic, M. (2009). Hypercoagulability in Cushing's syndrome: the role of specific 
haemostatic and fibrinolytic markers. Endocrine, 36(1), 70-74. https://doi.org/10.1007/s12020-0099186-y.

Lasta, C. S. (2011). Fatores de risco, parâmetros hematológicos, detecção molecular e sorológica de Ehrlichia canis e Anaplasma plantys em cães de porto Alegre/RS-Brasil. Universidade Federal do Rio Grande do Sul.

Mota, N. M., Ramaldes, F. M., \& Leal, D. R. (2019). Retrospective study of erliqiose canines cases. 1, $1-14$.

Peixoto, C. S. (2019). Alterações oculares e hematológicas em cães acometidos por Ehrlichia canis e co-infecções. Universidade de Brasília.

Pereira, B. J., Nunes, L. C., Martins Filho, S., \& Costa, F. S. (2011). The effects of prednisone therapy on dogs: a prospective study using ultrasonography, cytopathology and histopathology. Revista Ceres, 58(5), 561-566. https://doi.org/10.1590/S0034-737X2011000500005

Sá, R., Sá, I. S., Almeida, L. F., Miranda, G. S., Gomes, J. B., Santos, A. R. S. S., Silva, K. F. M., Araújo, M. S., Lisboa Neto, A. F. da S., Silva, J. C. F., Oliveira, M. A. L., Machado, F. C. F., \& Machado Júnior, A. A. N. (2018). Erliquiose canina: Relato de caso. Pubvet, 12(6), 1-6. https://doi.org/10.22256/pubvet.v12n6a118.1-6

Santos, M. A. M., Marçola, T. G., Mustafa, V. S., Medeiros, M., Tognoli, G. K., \& Volkweis, F. S. (2019). Estudo retrospectivo das alterações hematológicas nos casos de erliquiose em cães atendidos no distrito federal. Revista Científica de Medicina Veterinária Do UNICEPLAC, 5(1), 59-73.

Silva, B., Oliveira, F., Trombini, L., \& Regina, S. (2008). Exame da função hepática na medicina. Revista Investigação, 14(6), 17-21.

Silva, M., Costa, C., Cintra, C., Souza, G., Ribeiro, G., Heitor, T., Nascimento, M., Magalhães, L., \& Crivelenti, L. (2015). Alerações glomérulo-tubulares em cães infectados por Ehrlichia canis. Revista Investigação, 14(6), 17-21.

Sousa, V. R. F. (2006). Avaliação clínica, morfológica, hematológica, bioquímica e biomolecular de cães naturalmente infectados por Ehrlichia canis e Anaplasma platys. Universidade Federal Rural do Rio de Janeiro.

Souza, D. M. B., Coleto, Z. F., Souza, A. F., Silva, S. V, Andrade, J. K., \& Gimenez, G. C. (2012). Erliquiose transmitida aos cães pelo carrapato marrom (Rhipicephalus sanguineus). Ciência Veterinária Nos Trópicos, 15, 21-31.

Histórico do artigo:

Recebido: 17 de dezembro de 2020 Aprovado: 20 de janeiro de 2021. Disponível online: 30 de abril de 2021.
Licenciamento: Este artigoé publicado na modalidade Acesso Aberto sob a licença Creative Commons Atribuição 4.0 (CC-BY 4.0), a qual permite uso irrestrito, distribuição, reprodução em qualquer meio, desde que $\mathrm{o}$ autor e a fonte sejam devidamente creditados. 